\title{
Prospects for Atom Probe Tomography of Commercial Semiconductor Devices
}

\author{
D.J. Larson ${ }^{1,}$, D. Lawrence ${ }^{1}$, D. Olson ${ }^{1}$, T.J. Prosa ${ }^{1}$, D.A. Reinhard ${ }^{1}$, R.M. Ulfig ${ }^{1}$, P.H. Clifton ${ }^{1}$, \\ J.H. Bunton ${ }^{1}$, D. Lenz ${ }^{1}$, J.D. Olson ${ }^{1}$, L. Renaud ${ }^{2}$, I. Martin ${ }^{2}$ and T.F. Kelly ${ }^{1}$ \\ ${ }^{1}$ Cameca Instruments Inc., 5500 Nobel Drive, Madison, WI 53711 \\ ${ }^{2}$ Cameca SAS, 29 Quai des Gresillons, Gennevilliers 92230 FRANCE
}

For the nanoelectronics industries, atom probe tomography (APT) offers the prospect of unique capabilities for device characterization [1]. The advantages of APT for analysis of a 3D device include; a) three-dimensional compositional mapping of a relevant volume $\left(>10^{6} \mathrm{~nm}^{3}\right)$, b) high detection efficiency $(>50 \%)$, and c) high sensitivity (down to single atoms) for device analysis. The technique also has potential to be utilized as a composition standard for metrology applications. These advances have been made possible by recent major developments in instrumentation [2], specimen preparation [3], and often by methods correlation with TEM [4,5] and/or SIMS [5,6].

However, atom probe analyses of actual devices have been few and not generally from a fully packaged structure. This presentation will report progress made in APT analyses of individual transistors in off-the-shelf logic chips. A commercial 32-nm technology node device, an Intel i5-650 chip, was purchased at a retail outlet. Following depackaging, focused-ion-beam preparation [3] was used to create specimens. Fig. 1 shows a) trenches cut into the wafer, b\&c) the extracted coupon mounted on a microtip, d) a pre-tip cut from the coupon, and e-h) stages of focused ion beam annular sharpening [7] of the tip. Fig. 2 shows the feasibility of APT analysis with regions of SiGe (red), Fig. $2 \mathrm{~b}$, on either side of a high-k gate oxide (orange) being clearly visible. The channel region contains the dopant elements B, As, as well as C, Fig. 2b. Fig. 3 shows these elements as a concentration map where red is the maximum concentration and blue is a minimum (zero). These maps show evidence that the boron is spatially correlated to the Ge, the As in uniform and the carbon is clustering.

The field of view of the atom probe for this device is just adequate to observe portions of the source and drain on either side of the channel. In future technology nodes, the transistor characteristic lengths will be smaller and the present field of view achieved in this work will cover a larger portion of the components outside the channel. Although atom probe analysis of packaged devices is not currently routine, we expect that this approach to specimen preparation and analysis will mature quickly.

[1] D. J. Larson et al., "Atom Probe Tomography for Microelectronics”; in Handbook of Instrumentation and Techniques for Semiconductor Nanostructure Characterization, eds. R. Haight, F. Ross and J. Hannon, (World Scientific Publishing/Imperial College Press) 2011

[2] J. H. Bunton et al., Microsc. Microana., 13 (2007) 418.

[3] M. K. Miller et al., Microsc. Microanal. 13 (2007) 428.

[4] I. Arslan et al., Ultramicroscopy 108 (2008) 1579.

[5] K. Thompson et al., Science Sept. 7 (2007) 1370.

[6] P. Ronsheim, et al., Appl. Surf. Sci., 255 (2008) 1547.

[7] D. J. Larson et al., Ultramicroscopy 79 (1999) 287.

[8] J. Mardinly, 15 th Int. Conf. Micro. Semi. Materials (Springer Proc. Phys. vol. 120) (2007) 361. 


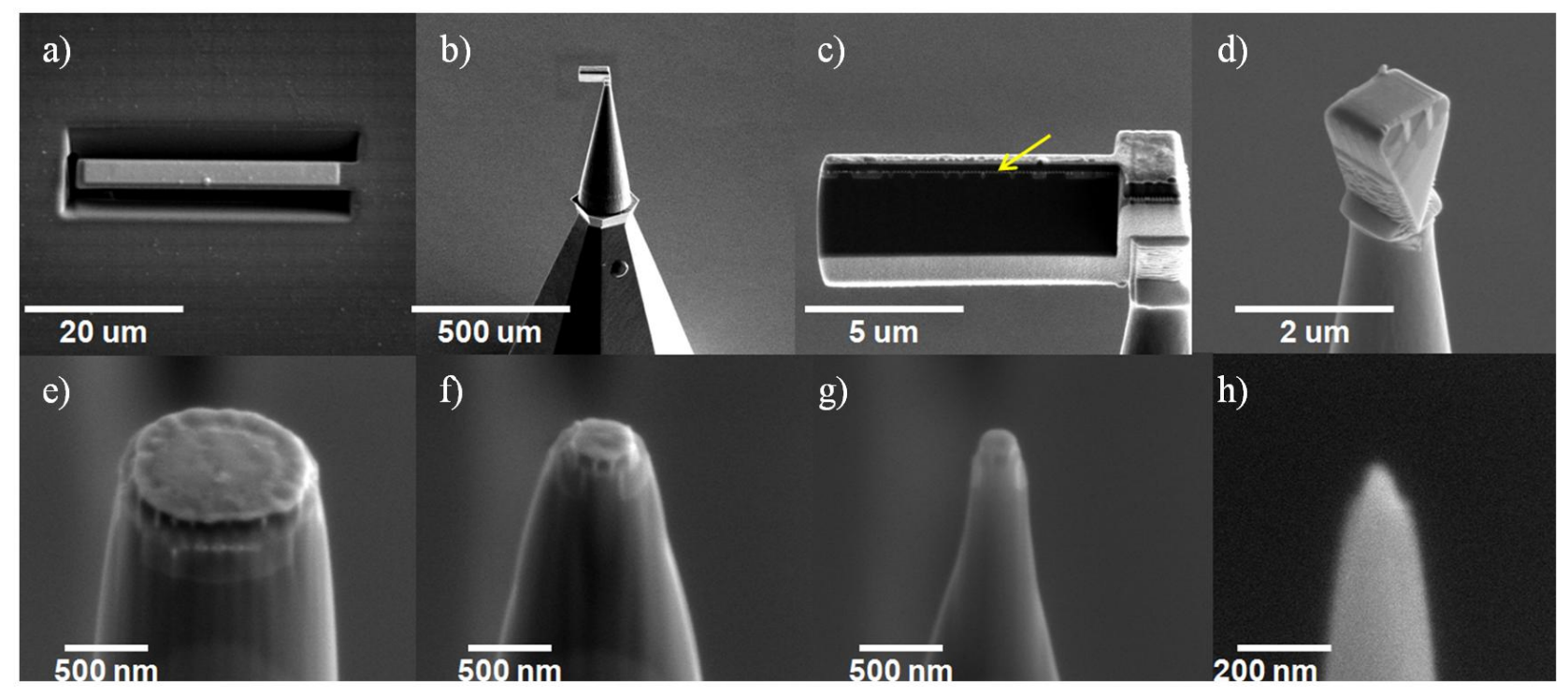

FIG. 1 Stages of focused ion beam specimen preparation from a) cutting trenches to h) final tip shape.

a) High-k + Metal Gate Transistor

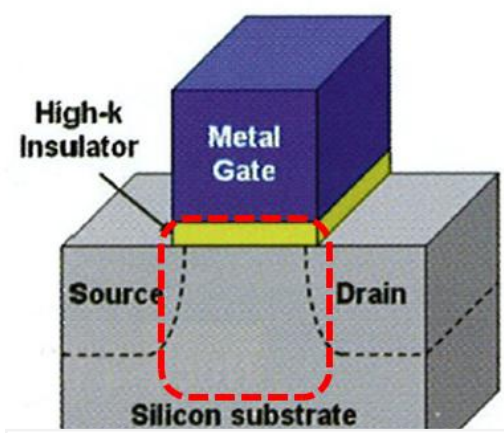

FIG. 2 a) Schematic of the transistor (from ref [8]) and b) atom maps from a $20 \mathrm{~nm}$ thick segment of the transistor.

\section{B $(0.025 \%)$}

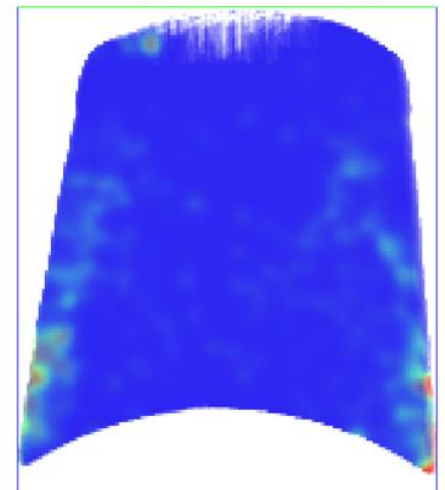

b)

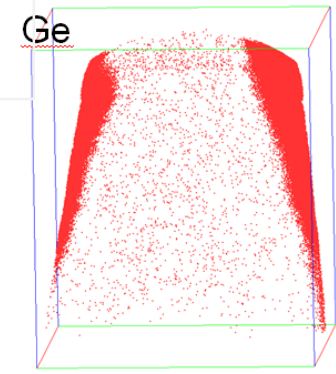

B

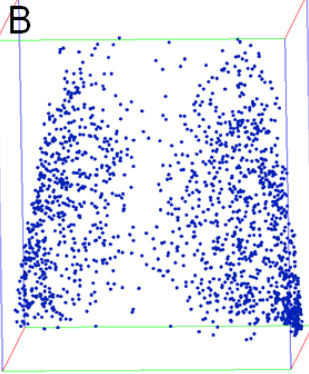

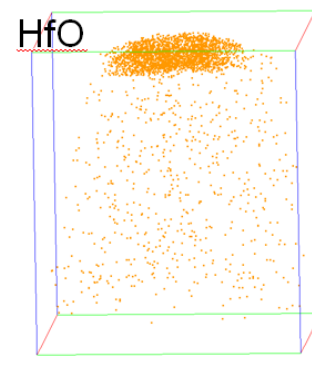

As

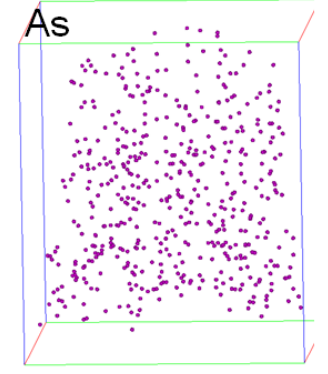

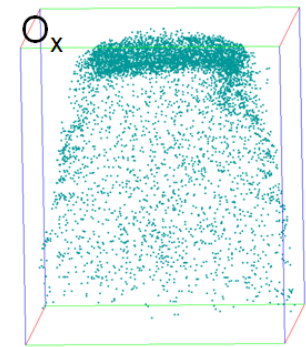

C

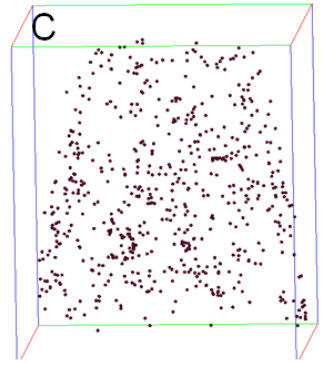

$\sim 70 \mathrm{~nm} \times 80 \mathrm{~nm}$ (viewed depth $70 \mathrm{~nm}$ )
As $(0.00025 \%)$

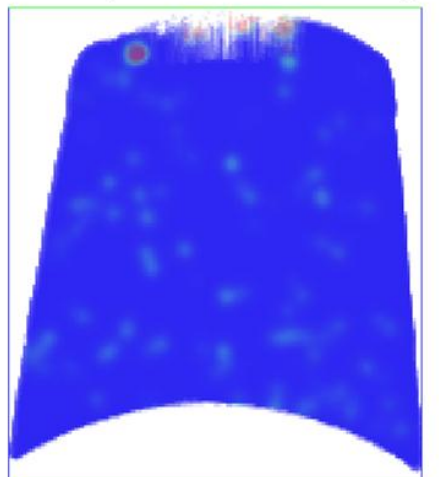

C $(0.5 \%)$

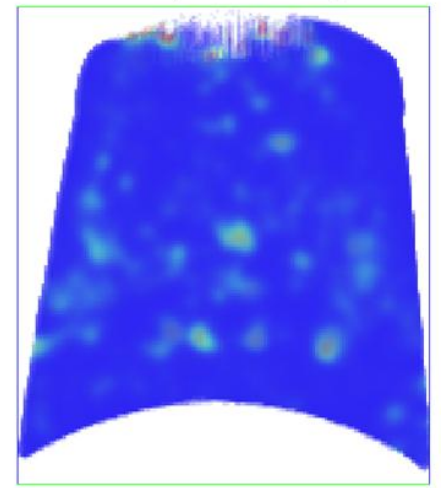

FIG. 3 Concentration maps showing clustering of dopants. The listed concentrations correspond to red in the colormap. 